\title{
Ф. КАПЛАНСКИИ
}

\section{О ДИФФУЗИИ КРУГОВОЙ ВИХРЕВОЙ НИТИ}

F. KAPLANSKI. UMARA KEERISNIIDI DIFUSIOONIST

$F$. KAPLANSKI. ON THE DIFFUSION OF. THE CIRCULAR VORTEX LINE

\section{(Представил И. Эпик)}

Рассматривается аналог классической задачи о диффузии прямолинейной вихревой нити с целью описания движения кольцевого вихря. Перенос завихренности б описывается уравнением

$$
\frac{\partial \zeta}{\partial t}+\frac{\partial}{\partial r}(v \zeta)+\frac{\partial}{\partial x}(u \zeta)=v^{*}\left[\frac{\partial^{2 \zeta}}{\partial x^{2}}+\frac{\partial^{2} \zeta}{\partial r^{2}}+\frac{q}{r} \frac{\partial \zeta}{\partial r}-\frac{q \zeta}{r^{2}}\right]
$$

которое при $q=1$ соответствует осесимметричной задаче, а при $q=0-$ плоской; $x, r-$ оси цилиндрической системы координат при $q=1$ или оси декартовой системы при $q=0$. Функция тока введена формулами

$$
u=\frac{1}{r^{q}} \frac{\partial \psi}{\partial r}-V(t), \quad v=-\frac{1}{r^{q}} \frac{\partial \psi}{\partial x}
$$

и может быть найдена как решение уравнения Пуассона

$$
-\zeta r^{q}=\frac{\partial^{2} \psi}{\partial x^{2}}+\frac{\partial^{2} \psi}{\partial r^{2}}-\frac{q}{r} \frac{\partial \psi}{\partial r} .
$$

Здесь $V(t)$ - скорость вихревого кольца, равная $\frac{d x_{0}(t)}{d t}$, где $x_{0}(t)-$ пройденное вихрем расстояние.

Начальное условие определяется условиями образования вихревого кольца или вихревой пары: при $t=0$ функция $\zeta(r, x, 0)$ равна нулю всюду, кроме точки $r=a$, где она бесконечна (круговая вихревая нить радиуса $a$ при $q=1$ или две противоположно направленных прямолинейных вихревых нити на расстоянии $2 a$ при $q=0$ ).

Краевые условия

$$
\begin{array}{lll}
\zeta=\psi=0 & \text { при } & r=0 \quad \text { (условие симметрии), } \\
\zeta, \psi \rightarrow 0 & \text { при } & x^{2}+r^{2} \rightarrow \infty .
\end{array}
$$

Интегрируя (1) при условиях (3) получим условие сохранения

$$
\frac{(2 \pi)^{q}}{2} \int_{-\infty}^{\infty} \int_{0}^{\infty} r^{q+1 \zeta}(r, x, t) d x d r=B .
$$

Рассматриваются две модели.

I. Ламинарное вихревое кольцо, когда $v^{*}=v-$ коэффициент молекулярной вязкости. 
II. Турбулентное вихревое кольцо, когда $v^{*}=v_{*} t^{-\frac{1}{3-q}}$, где $v_{*}$ 一 постоянная величина, которая должна определяться сравнением результатов расчета и эксперимента. Здесь зависимость от времени для коэффициента турбулентной вязкости, предложенная для стадии автомодельного развития вихря [ $\left.{ }^{1}\right]$, формально распространена и на начальную стадию его развития.

В развитие результатов А. Калтаева $\left[{ }^{2}\right]$ выпишем точное решение осесимметричной и плоской задачи для I и II моделей в пренебрежении конвекцией относительно движущегося вихря (решение найдено методом разделения переменных)

$$
\zeta=A t^{-\alpha} \mathrm{e}^{-\frac{\left(x-x_{0}(t)\right)^{2}+r^{2}+a^{2}}{2 p}} \cdot f\left(\frac{r a}{p}\right)
$$

Выражения $\alpha, p, f$ и $A$ приведены в таблице.

\begin{tabular}{c|c|c|l|l|l}
\hline$q$ & Модель & $\alpha$ & $p$ & $f$ & $A$ \\
\hline 0 & I & 1 & $2 v t$ & $s h(m)$ & $B / 4 \pi v a$ \\
0 & II & $2 / 3$ & $3 v \cdot t^{2 / 3}$ & $s h(m)$ & $B / 6 \pi v_{*} a$ \\
1 & I & $3 / 2$ & $2 v t$ & $I_{1}(m)$ & $B / 4(\pi v)^{3 / 2 a}$ \\
1 & II & $3 / 4$ & $4 v_{*} t^{1 / 2}$ & $I_{1}(m)$ & $B /\left(2 \pi v_{*}\right)^{3 / 2} a$
\end{tabular}

Здесь $I_{1}-$ модифицированная функция Бесселя, $A$ определяется условием нормировки (4). При малых значениях $\frac{a}{\sqrt{p}}$ для $r \leqslant c a(c-$ любое конечное число), используя разложения в ряды $I_{1}\left(\frac{r a}{p}\right)$ и $\mathrm{e}^{-\frac{a^{2}}{2 p}}$, получим приближение (5), совпадающее с ранее найденными автомодельными решениями $\left.{ }^{3-5}\right]$

$$
\zeta=A t^{-\alpha} \mathrm{e}^{-\frac{\left(x-x_{0}(t)\right)^{2}+r^{2}}{2 p}}\left(\frac{r a}{4 p}+0\left(c\left(\frac{a^{4}}{8 p^{2}}\right)\right)\right) .
$$

При больших значениях $\frac{a}{\sqrt{p}}$ для $r \geqslant \frac{a}{c}$ при использовании асимптотического представления $I_{1}\left(\frac{r a}{p}\right)$ имеем другое приближение (5)

$$
\zeta=A t^{\alpha} \mathrm{e}^{-\frac{\left(x-x_{0}(t)\right)^{2}+(r-a)^{2}}{2 p}} \sqrt{\frac{p}{2 \pi r a}}\left(1+0\left(\frac{c p}{a^{2}}\right)\right) .
$$

Радиальные координаты характерной точки максимума вихря из (6) и (7) определяются по формулам

$$
\begin{gathered}
r_{m}=\sqrt{p} \\
r_{m}=\frac{a}{2}+\sqrt{\frac{a^{2}}{4}-\frac{p}{2}}
\end{gathered}
$$

Таким образом, в явлении диффузии круговой вихревой нити в безграничной жидкости можно выделить две стадии, описываемые выраже- 


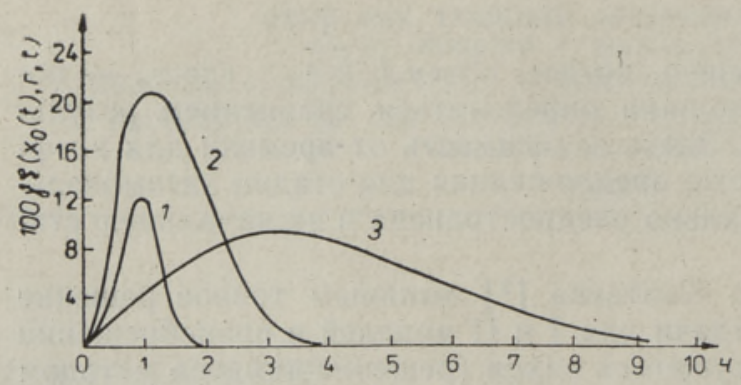

Изменение линейного решения с течением времени. $1-t=0,1$, $\gamma=10^{-2 / 3}$ (описывается приближением (6)); $2-t=1$, $\gamma=1 ; \quad 3-t=10, \quad \gamma=10^{-2 / 3}$ (описываются приближением (7); модель I, $q=1, v=0,5)$.

ниями (7) и (6). В первой из них резко выраженная тороидальная структура вихря развивается неавтомодельно. Во второй стадии происходит автомодельное развитие формы вихря при потере тороидальности. Аналогичная ситуация сохраняется и для плоской задачи. Переход вихря из первой стадии во вторую (изменение распределения завихренности с увеличением параметра $\left.\frac{a}{\sqrt{p}}\right)$ иллюстрирует рисунок. Концентрированное распределение завихренности (кривая 1) согласуется с единственным детальным экспериментальным исследованием кольцевого вихря при использовании лазерного доплеровского измерителя, определяющего две компоненты скорости [ $\left.{ }^{6}\right]$. Отметим, что найденное решение (5) является решением полной задачи (1)-(4) при $\frac{B}{v^{2} t} \rightarrow 0$ для первой модели $\left[{ }^{3}\right]$ и при $v_{*} \rightarrow \infty$ для второй модели $\left[{ }^{5}\right]$.

\section{ЛИТЕРАТ У РА}

1. Луговцов А. А., Луговцов Б. А., Тарасов В. Ф. Динамика сплошной среды, вып. III, $50-60$ (1969).

2. Калтаев А. В кн.: Динамика сплошной ореды. Алма-Ата, КазГУ, 1982, 63-70.

3. Ершин Ш. А., Калтаев А. В кн.: Прикладная механика. Применение математических методов в естествознании. Алма-Ата, КазГУ, 1979, 164-169.

4. Луговцов Б. А. В кн.: Некоторые проблемы математики и механики. М., «Наука», $1970,182-189$.

5. Капланский Ф., ఫпитейн А. Изв. АН ЭССР. Физ. Матем., 22, № 4, 386-393 (1973).

6. Sulivan, J. P., Widnall, S. E. AIAA Journal, 11, № 10, 1384-1389 (1973).

Институт термофизики и электрофизики Академии наук Эстонской ССР
Поступила в редакцию 7/IX 1983 